\title{
Synthesis and Glycosylation of 2'-(Benzyloxycarbonyl)benzyl Glycosides as Glycosyl Donors
}

\author{
Kwan Soo Kim, Moon Eui Lee, and Jin Won Cho" \\ Center for Bioactive Molecular Hybrids and Deparment of Chemistry and \\ ${ }^{\dagger}$ Department of Biologn; Yonsei University; Seoul 120-749. Korea \\ Recen'ed October 23, 2003
}

Key Words : 2'-(Benzyloxycarbonyl)benzyl glycosides, Glycosylation, Glycosyl donor

Recent advances in glycobiology have directed an increased attention to efficient and stereoselective glycosylation of oligosaccharides.' Devising new glycosyl donors and developing new activation systems for existing donors have led to major advances in this field. Nevertheless, there still remains a need for more efficient and generally applicable new glycosyl donors although several glycosyl donors are presently available. ${ }^{2}$ In fact, there have been recent reports on new glycosyl donors and new activation systems. ${ }^{3}$ We have also recently reported a novel type of glycosyl donors, the 2'-carboxybenzyl (CB) glycoside $\mathbf{B}$, as shown in Figure 1 for stereoselective $\beta$-mannopyranosylation and 2-deoxyglycosylation and applied this methodology to the synthesis of a tetrasaccharide. ${ }^{6}$ The CB glycoside $B$ was prepared from the 2'-(benzyloxycarbonyl)benzyl (BCB) glycoside A by the selective removal of its benzyl ester functionality." Treatment of the $C B$ glycoside $B$ with triflic anhydride followed by spontaneous lactonization of the resulting glycosyl triflate $\mathbf{C}$ afforded the oxocarbenium ion $\mathbf{D}$ by extrusion of stable phthalide. Reaction of the oxocarbenium jon D with the glycosyl acceptor (Sugar-OH) gave the desired glycoside $\mathbf{F}$ as shown in Figure 1. The direct generation of the oxocarbenium ion $\mathbf{D}$ from the $\mathrm{BCB}$ glycoside $\mathbf{A}$ would be more convenient than that from the $\mathrm{CB}$ glycoside $\mathbf{B}$ through the triflate $\mathbf{C}$. Thus, we envisaged that $\mathrm{I}$ ewis acidmediated lactonization of the BCB glycoside A would liberate stable phthalide to generate the oxocarbenium ion D as shown in Figure 1. Herein we report the glycosylation of the BCB glycoside A with glycosyl acceptors in the presence of TMSOTf as a promoter.

Coupling of the tetrabenzoylglucosyl bromide $\mathbf{I}^{7}$ and benzyl 2-(hydroxymethyl)benzoate $(2)^{k}$ in the presence of mercury salts at $0{ }^{\circ} \mathrm{C}$ in acetonitrile gave the $\mathrm{BCB}$ tetrabenzoylglucoside 3 in $84 \%$ yield. The BCB tetrabenzylglucoside 5 , on the other hand, was prepared by the benzylation of the known BCB glucopyranoside 4 as shown in Scheme 1. The BCB 2.3-di-O-benzoylcyclohexylideneglucoside 7 was also prepared from the compound 4 by the two-step sequence: (i) selective cyclohexylidenation of the compound 4 with 1,1-dimethoxycyclohexane in the presence of $p-\mathrm{TsOH}$ to afford the diol 6 in $82 \%$ yield and (ii) benzoylation of the resulting diol 6 with benzoyl chloride to give the compound 7 in $90 \%$ yield as shown in Scheme 1.

Glycosylation of the BCB tetrabenzoylglucoside 3 with the glycosyl acceptor $\mathbf{8}$ was carried out in acetonitrile by addition of TMSOTf at $0{ }^{\circ} \mathrm{C}$ and allowing the reaction mixture to warm over $3 \mathrm{~h}$ to room temperature to afford only the $\beta$-disaccharide 9 in $71 \%$ yield as shown in Scheme 2 . The fact that the $\beta$-disaccharide 9 was obtained exclusively without formation the $\alpha$-disaccharide indicates that the participating group at $\mathrm{C}-2$ is working well in the glycosylation with the BCB glycoside. Trimethylsilyl triflate was found to be a good promoter but other lewis acids, such as $\mathrm{BF}_{3} \cdot \mathrm{OF}_{2}$ and $\mathrm{SnCl}_{4}$, did not activate the glycosyl donor 3 . The same glycosylation reaction in methylene chloride instead of acetonitrile resulted in a little lower yield of the disaccharide 9 while $\mathrm{E}_{2}, \mathrm{O}$ and THF were found to be not proper solvents for the present glycosylation. The "armed' BCB tetrabenzylglucoside 5 was a more reactive glycosyl donor than the "disamed" BCB tetrabenzoylglucoside 3 as expected ${ }^{8}$ and the glycosylation of 5 proceeded at lower temperature than that of 3 . Thus, glycosylation of the

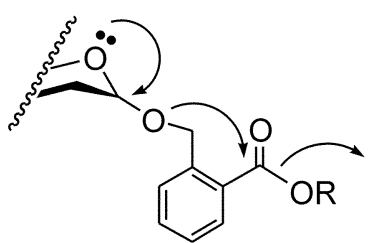
A $\mathrm{R}=\mathrm{Bn}$
B $\mathrm{R}=\mathrm{H}$
C $R=T f$

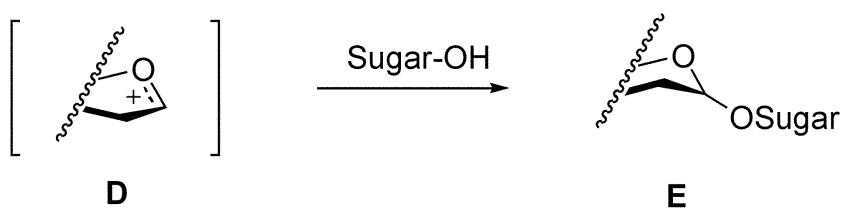

Figure 1

\footnotetext{
"lo whom correspondence should be addressed. E-mail: kwan $(\bar{a})$ yonsei.ac.kr
} 


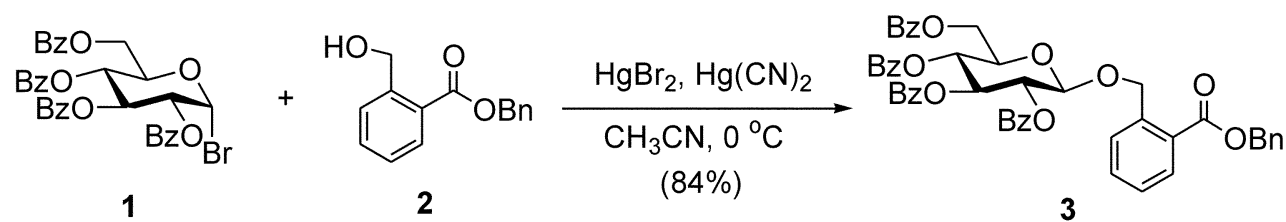<smiles>O=C(OCc1ccccc1COC1OC(CO)C(O)C(O)C1O)c1ccccc1Br</smiles>

4

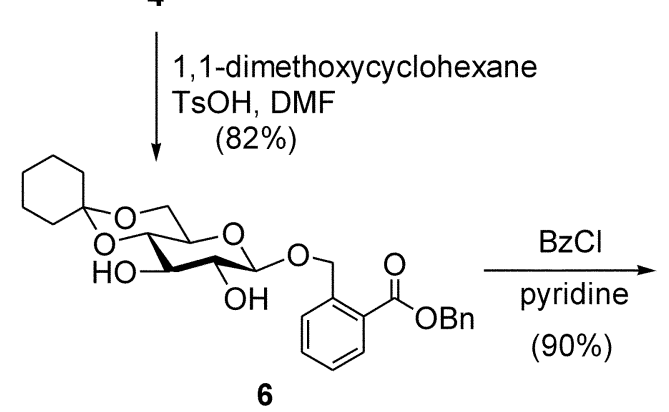

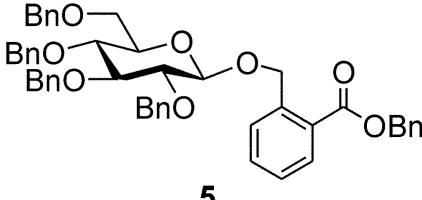

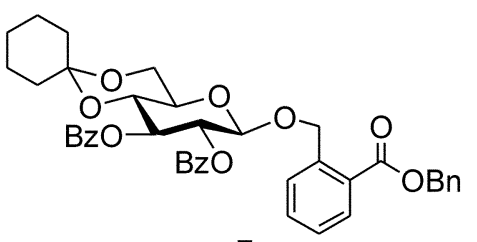

7

Scheme 1

glycosyl donor 5 with the glycosyl acceptor 8 at $-25^{\circ} \mathrm{C}$ in the presence of TMSOlf in aceronitrile provided a separable mixture of disaccharides $10 \alpha$ and $10 \beta(1: 3)$ in $78 \%$ yield. On the other hand, the $\mathrm{BCB} 4,6$-cyclohexylideneglucoside 7 showed intermediate reactivity in the glycosylation. Glycosylation of the glycosyl donor 7 with the acceptor 8 in the presence of 1 MSO [ $\mathrm{f}$ in acetonitrile proceeded at $0{ }^{\circ} \mathrm{C}$ to afford only $\beta$-disaccharide 11 in $73 \%$ yield.

In sumınary, $\mathrm{BCB}$ glycosides, which had been used as the precursor to a novel type of glycosyl donors, $\mathrm{CB}$ glycosides, were also found to be good glycosyl donors. Glycosylation of $\mathrm{BCB}$ glycosides with glycosyl acceptors employing [MSO]f as a promoter readily afforded disaccharides.

\section{Experimental Section}

Synthesis of 2-(Benzyloxycarbonyl)benzyl 2,3,4,6-tetra$\boldsymbol{O}$-benzoyl- $\boldsymbol{\beta}$-D-glucopyranoside (3). To a stirred solution of 2,3,4,6-tetra- $O$-benzoyl- $\alpha$-D-glucopyranosyl bromide $(1)^{7}$ (1.86 g, $2.8 \mathrm{mmol}$ ) and benzyl 2-(hydroxymethyl)-

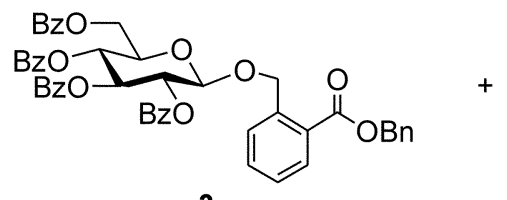

3<smiles>O=C(OCc1ccccc1)c1ccccc1COC(OCCOc1ccccc1)C(Br)(Br)COc1ccccc1</smiles>

5

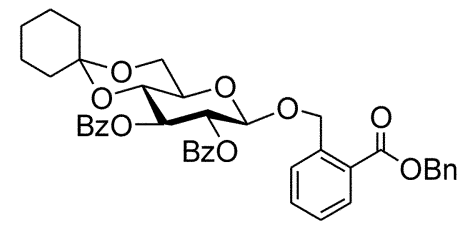

7

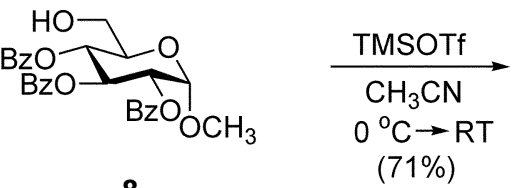

8

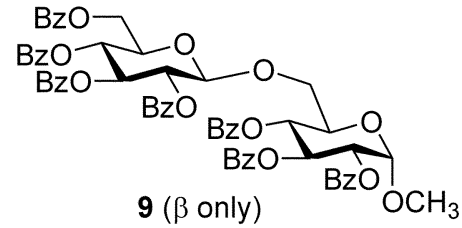

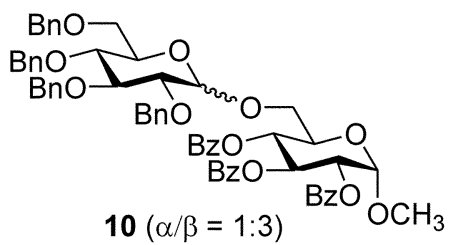

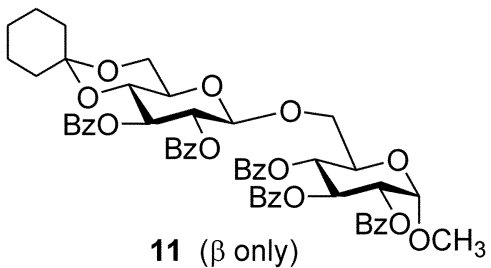

Scheme 2 
benzoate $(2)^{4}\left(0.82\right.$ g. 3.4 munol, 1.2 equiv) in $\mathrm{CH}_{3} \mathrm{CN}(5$ $\mathrm{mL}$ ) in the presence of $+\mathrm{A}$ molecular sieves were added mercury (II) bromide (1.22 g, $3.4 \mathrm{~mm}$ ol. 1.2 equiv) and mercury (II) cyanide (0.85 g, 3.4 numol, 1.2 equiv) at $0^{\circ} \mathrm{C}$. After stirring at $0^{\circ} \mathrm{C}$ for further $10 \mathrm{~min}$, the reaction mixture was filtered and the filtrate was concentrated. The resulting oil was dissolved in EtOAc $(50 \mathrm{~mL})$ and the solution was washed with saturated aqueous $\mathrm{NaHCO}_{3}(2 \times 50 \mathrm{~mL})$ and brine $(50 \mathrm{~mL})$. The organic phase was dried $\left(\mathrm{MgSO}_{4}\right)$ and concentrated in vacuo and the residue was purified by silica gel flash column chromatography ( $n$-hexane/EtOAc. 2 : 1. $v / v)$ to afford the compound $3(1.94 \mathrm{~g}, 84 \%)$ as white solids: mp 47-50 ${ }^{\circ} \mathrm{C}: \mathrm{R}_{f}=0.45$ (n-hexane/EtOAc. $\left.2: \mathrm{l} . \mathrm{v} / \mathrm{v}\right):[\alpha]_{\mathrm{D}}^{20}$ $=+9.6\left(\mathrm{c}=2.7 . \mathrm{CHCl}_{3}\right) ;{ }^{1} \mathrm{H}$ NMR $\left(250 \mathrm{MHz} . \mathrm{CDCl}_{3}\right) \delta$ $4.11-4.18$ (m. IH). 4.49 (dd, $J=5.2,12.2 \mathrm{~Hz}, 1 \mathrm{H}$ ), 4.65 (dd. $J=3.1 .12 .1 \mathrm{~Hz}, \mathrm{IH}) .4 .97(\mathrm{~d}, J=7.8 \mathrm{~Hz}, \mathrm{IH}), 5.19$ (d. $J=$ $14.7 \mathrm{~Hz} .1 \mathrm{H}), 5.2 \mathrm{l}$ (s. $2 \mathrm{H}), 5.34$ (d, $J=14.7 \mathrm{~Hz}, \mathrm{lH}) .5 .65-$ 5.77 (n. 2H). 5.92 (t. $J=9.6 \mathrm{~Hz} . \mathrm{HH}) .7 .21-8.05$ (m, 29H): ${ }^{13} \mathrm{C} \mathrm{NMR}\left(63 \mathrm{MHz}, \mathrm{CDCl}_{3}\right) \delta$ 63.1. 66.7, 69.5. 69.9. 72.1. 72.4, 73.1. 100.9. 127.2. 127.5, 127.7, 128.3, 128.4. 128.5. 128.7. 128.9, 129.4, 129.8. 129.9. 130.7, 132.7. 133.2. 139.8. 165.3(2), 165.9, 166.5; IR (NaCl) 1256. 1453. 1604. $1736 \mathrm{~cm}^{-1}$. Anal. Calcd for $\mathrm{C}_{419} \mathrm{H}_{41} \mathrm{O}_{13}: \mathrm{C}, 71.70 ; \mathrm{H} .4 .91$. Found: C. $71.67: \mathrm{H}, 4.83$

2-(Benzyloxycarbonyl)benzyl 2,3,4,6-Tetra-O-benzy]B-D-glucopyranoside (5). To a solution of 2-(benzyloxycarbonyl)benzyl- $\beta$-D-glucopyranoside $(4)^{4}$ (3.28 g. 8.1 mnol. 1.0 equiv) and benzyl bromide $(3.87 \mathrm{~mL} .32 .5 \mathrm{~nm}$ ol. 4.0 equiv) in DMF (30 mL) was added sodium hydride $(1.3$ g. 32.5 mumol, 4.0 equiv) at $0^{\circ} \mathrm{C}$ and then the ice bath was removed. After stiring at room temperature for $\mathrm{l} \mathrm{h}$. the reaction mixture was quenched with water $(50 \mathrm{~mL})$ and extracted with EtOAc $(2 \times 100 \mathrm{~mL})$. The combined organic layer was washed with saturated aqueous $\mathrm{NH}_{4} \mathrm{Cl}(50 \mathrm{~mL})$ and brine $(50 \mathrm{~mL})$. dried $\left(\mathrm{MgSO}_{4}\right)$. and concentrated in vacuo. The residue was purified by silica gel flash columm chromatography ( $n$-hexane/EtOAc. $4: 1 . \mathrm{v} / \mathrm{v}$ ) to afford the compound $5(7.48$ g. $85 \%): \mathrm{R}_{f}=0.50$ (n-hexane/EtOAc. $4: 1, v / v):[\alpha]_{D}^{20}=-1.72\left(\mathrm{c}=3.3, \mathrm{CHCl}_{\mathrm{j}}\right):{ }^{\mathrm{l}} \mathrm{H}$ NMR $(250$ $\left.\mathrm{MHz} \mathrm{CDCl}_{3}\right) \delta 3.43-3.46(\mathrm{~m}, \mathrm{lH}), 3.55-3.74(\mathrm{~m}, 5 \mathrm{H}), 4.50-$ $4.65(\mathrm{~m} .4 \mathrm{H}) .4 .75-4.85(\mathrm{~m} .3 \mathrm{H}) .4 .93-5.02(\mathrm{~m} .2 \mathrm{H}) .5 .15(\mathrm{~d}$. $J=14.8 \mathrm{~Hz}, 1 \mathrm{H}) .5 .30$ (s. $2 \mathrm{H}), 5.40$ (d. $J=14.8 \mathrm{~Hz}, 1 \mathrm{H})$. $7.15-7.48(\mathrm{~m}, 27 \mathrm{H}), 7.79(\mathrm{~d}, J=7.7 \mathrm{~Hz}, 1 \mathrm{H}), 8.03(\mathrm{dd}, J=$ 13. $7.8 \mathrm{~Hz}, 1 \mathrm{H}):{ }^{13} \mathrm{C}$ NMR $\left(63 \mathrm{MHz} . \mathrm{CDCl}_{3}\right) \delta 66.8 .68 .8$. $69.4,73.6,75.1(2), 75.8,78.0,82.5,84.9,103.2,127.1$. $\begin{array}{lllllll}127.6 & 127.7(2), & 127.8, & 127.9 & 128.0(2), & 128.1, & 128.2 .\end{array}$ 128.4(2). 128.5(2), 128.7. 130.8, 132.7. 136.0.138.2. 138.5. 138.8. 140.8. 166.7: IR ( $\mathrm{CHCl}_{j}$ film) $1077.1266 .1729 \mathrm{~cm}^{-1}$. Anal. Calcd for $\mathrm{C}_{413} \mathrm{H}_{48} \mathrm{O}_{8}$ : C. 76.94: H. 6.33. Found: C. 76.98: H. 6.35.

2-(Benzyloxycarbonyl)benzyl 4,6-O-CyclohexylideneB-D-glucopyranoside (6). A solution of 2-(benzylosycarbonỵl)benzyl- $\beta$-D-glucopyranoside $(\mathbf{4})^{4}$ (934 mg. 2.31 mmol. 1.0 equiv) 1. l-dimethoxycyclohexane (666 mg. 4.62 mmol. 2.0 equiv), and $p$-TsOH ( $8 \mathrm{mg} .0 .46 \mathrm{mmol} .0 .2$ equiv) in DMF $(10 \mathrm{~mL})$ was stirred at $60^{\circ} \mathrm{C}$ for $4 \mathrm{~h}$. The reaction mixture was quenched with saturated aqueous $\mathrm{NaHCO}_{3}$ (30
$\mathrm{mL})$, and extracted with EtOAc $(3 \times 30 \mathrm{~mL})$. The combined organic layer was washed with saturated aqueous $\mathrm{NH}_{4} \mathrm{Cl}(2$ $\times 30 \mathrm{~mL})$ and brine $(30 \mathrm{~mL})$. dried $\left(\mathrm{MgSO}_{4}\right)$, and concentrated in vacto. The residue was purified by silica gel flash column chromatography ( $n$-hexane/EtOAc. $1: 2, v / v)$ to afford the compound $6(917 \mathrm{mg} .82 \%$ ) as white solids: $\mathrm{mp}$ $58-60{ }^{\circ} \mathrm{C}: \mathrm{R}_{f}=0.6(n$-hexane/EtOAc. $1: 2 . \mathrm{v} / \mathrm{v}):[\alpha]_{\mathrm{D}}^{20}=$ $-34.9\left(\mathrm{c}=1.3 . \mathrm{CHCl}_{3}\right) ;{ }^{1} \mathrm{H}$ NMR $\left(250 \mathrm{MHz} . \mathrm{CDCl}_{2}\right) \delta 1.43-$ 2.02 (m. loH). 2.87 (s. lH), 3.06 (s. IH). 3.22-3.32 (m. lH), $3.48-3.81$ (m. 5H), 3.91 (dd, $J=5.5,10.7 \mathrm{~Hz} .1 \mathrm{H}), 4.78$ (d. $J$ $=7.6 \mathrm{~Hz}, 1 \mathrm{H}), 5.09(\mathrm{~d}, J=13.0 \mathrm{~Hz}, \mathrm{lH}), 5.19(\mathrm{~d}, J=13.0$ Hz. lH), 5.34 (s, lH). 7.32-7.64 (m, 8H). 7.99 (dd, $J=1.1$, $7.7 \mathrm{~Hz} .1 \mathrm{H}){ }^{13} \mathrm{C}$ NMR $\left(63 \mathrm{MHz} . \mathrm{CDCl}_{3}\right) \delta 22.7,22.9,25.7$, $27.9,38.0$. 61.5. 67.1. 67.7. 70.2, 72.4, 73.9, 74.8. 100.0. 103.1. 127.9, 128.3. 128.4. 128.7, 128.8, 129.0. 130.9 . $132.7,135.9,139.4 .167 ;$ IR $\left(\mathrm{CHCl}_{2}\right.$ film $) 1084.1269 .1729$ $\mathrm{cm}^{-1}$. Anal. Calcd for $\mathrm{C}_{27} \mathrm{H}_{32} \mathrm{O}_{8}: \mathrm{C}, 66.93: \mathrm{H}$. 6.66. Found: C. 66.93: H, 6.68.

2-(Benzyloxycarbonyl)benzyl 2,3-Dj- $O$-benzoyl-4,6- $O$ cyclohexylidene- $\beta$-D-glucopyranoside (7). A solution of 2 (benzyloxycarbonyl)benzyl 4.6-O-cyclohexylidene- $\beta$-Dglucopyranoside (6) (400 $\mathrm{mg}, 0.83 \mathrm{mmol} .1 .0$ equiv) and benzoyl chloride (350 $\mathrm{mg}, 2.49 \mathrm{mmol}$. 3.0 equiv) in pyridine $(5 \mathrm{~mL})$ was stirred at room temperature for $2 \mathrm{l}$. The reaction mixture was quenched with saturated aqueous $\mathrm{NH}_{4} \mathrm{Cl}$ (10 $\mathrm{mL})$, and extracted with EtOAc $(3 \times 15 \mathrm{~mL})$. The combined organic layer was washed with saturated aqueous $\mathrm{NH}_{4} \mathrm{Cl}(2$ $\times 15 \mathrm{~mL})$ and brine $(15 \mathrm{~mL})$, dried $\left(\mathrm{MgSO}_{4}\right)$. and concentrated in vacuo. The residue was purified by silica gel flash column chromatography ( $n$-hexane/EtOAc. $2: 1, v / v)$ to afford the compound $7(516 \mathrm{mg} .90 \%$ ) as white solids: $\mathrm{mp}$ $66-68{ }^{\circ} \mathrm{C}: \mathrm{R}_{f}=0.6$ (n-hexane/EtOAc. $\left.2: 1 . v / v\right):[\alpha]_{\mathrm{D}}^{14}=$ $+15.6\left(\mathrm{c}=1.0, \mathrm{CHCl}_{2}\right) ;{ }^{1} \mathrm{H} \mathrm{NMR}\left(250 \mathrm{MHz}, \mathrm{CDCl}_{2}\right) \delta 1.27-$ 2.09 (m. l0H). 3.48-3.58 (m, IH). 3.85-4.06 (m, 3H). 4.87 (d. $J=7.3 \mathrm{~Hz} . \mathrm{lH}) .5 .16$ (d. $J=15.0 \mathrm{~Hz}, 1 \mathrm{H}), 5.25$ (s. $2 \mathrm{H})$, 5.33 (d. $J=15.0 \mathrm{~Hz} . \mathrm{lH}), 5.50-5.68$ (m. $2 \mathrm{H}$ ), $7.15-7.99$ (m, $19 \mathrm{H}):{ }^{13} \mathrm{C}$ NMR $\left(63 \mathrm{MHz}, \mathrm{CDCl}_{3}\right) \delta 22.5 .22 .7,25.5,27.6$. $37.8 .61 .5 .66 .7 .67 .7,69.5,71.1,72.7(2) .99 .9 .101 .4,127.0$. $127.2,127.3,127.8,128.2,128.4,128.7,129.5,129.6$. $129.7,129.9(2), 130.6,132.7,133.1,133.2,135.9,140.1$ 165.3. 165.7. 166.4: IR ( $\mathrm{CHCl}_{3}$ film $) 1111,1275,1407$. 1637. $1743 \mathrm{~cm}^{-1}$. Anal. Calcd for $\mathrm{C}_{41} \mathrm{H}_{411} \mathrm{O}_{101:} \mathrm{C} .71 .08: \mathrm{H}$. 5.82. Found: C. 71.06: H. 5.79

Methyl (2,3,4,6-Tetra- $O$-benzoyl- $\beta$-D-glucopyranosyl)$(1 \rightarrow 6)-2,3,4$-tri- $O$-benzoyl- $\alpha$-D-glucopyranoside $(9) .^{\circ} \mathrm{A}$ solution of 2-(benzy loxycarbonyl)benzyl 2,3.4.6-tetra-Obenzoyl- $\beta$-D-glucopyranoside (3) $(31 \mathrm{mg} .0 .038 \mathrm{mmol}, 1.0$ equiv). methyl 2.3.4-tri- $O$-benzoyl- $\alpha$-D-glucopyranoside (8) (59 mg. $0.11 \mathrm{mmol}, 3.0$ equiv) and TMSOTf (42 mg. 0.19 mumol. 5.0 equiv.) in $\mathrm{CH}_{3} \mathrm{CN}(3 \mathrm{~mL})$ was stirred at $0^{\circ} \mathrm{C}$ for $10 \mathrm{~min}$. and allowed to warm over $3 \mathrm{~h}$ to room temperature. The reaction mixture was quenched by addition of saturated aqueous $\mathrm{NaHCO}_{3}$ solution $(2 \mathrm{~mL})$ and then extracted with $\mathrm{CH}_{2} \mathrm{Cl}_{2}(3 \times 15 \mathrm{~mL})$. The combined organic layer was washed with brine $(15 \mathrm{~mL})$, dried $\left(\mathrm{MgSO}_{4}\right)$, and concentrated in vacuo. The residue was purified by silica gel flash colunu chromatography (toluene/EtOAc. $10: 1, \mathrm{v} / \mathrm{v}$ ) to 
afford the $\beta$-disaccharide $9(29 \mathrm{mg}, 71 \%$ ) as white solids: Inp 91-93 ${ }^{\circ} \mathrm{C}: \mathrm{R}_{f}=0.30$ (toluene/EtOAc, $10: 1, \mathrm{v} / \mathrm{v}$ ): $[\alpha]_{D}^{20}=$ $+39.0\left(\mathrm{c}=4.0 . \mathrm{CHCl}_{3}\right),{ }^{1} \mathrm{H}$ NMR $\left(500 \mathrm{MHz} . \mathrm{CDCl}_{3}\right) \delta 3.11$ $(\mathrm{s} .3 \mathrm{H}) .3 .78(\mathrm{dd}, J=7.5 .11 .5 \mathrm{~Hz} .1 \mathrm{H}), 4.08-4.16(\mathrm{~m}, 2 \mathrm{H})$. $4.20-4.24$ (m. IH). 4.45 (dd, $J=5.5,12.0 \mathrm{~Hz}, 1 \mathrm{H}), 4.60$ (dd. $J=3.0 .12 .0 \mathrm{~Hz}, \mathrm{IH}) .4 .94(\mathrm{~d}, J=3.5 \mathrm{~Hz}, \mathrm{IH}) .4 .97(\mathrm{~d} . J=$ $8.0 \mathrm{~Hz}, \mathrm{lH}) .5 .09$ (dd. $J=3.5 .10 .0 \mathrm{~Hz} .1 \mathrm{H}) .5 .31(\mathrm{t} . J=10.0$ $\mathrm{Hz}, \mathrm{IH}) .5 .56(\mathrm{dd}, J=8.5 .18 .5 \mathrm{~Hz} . \mathrm{lH}), 5.65(\mathrm{t} . J=10.0 \mathrm{~Hz}$. $\mathrm{lH}), 5.59(\mathrm{t}, J=9.5 \mathrm{~Hz} . \mathrm{lH}), 6.07(\mathrm{t}, J=10.0 \mathrm{~Hz} . \mathrm{lH}), 7.23-$ $8.00(\mathrm{~m}, 35 \mathrm{H}):{ }^{13} \mathrm{C} \mathrm{NMR}\left(75 \mathrm{MHz}, \mathrm{CDCl}_{\hat{3}}\right) \delta$ 55.2. 63.2. $68.8,69.1 .69 .9(2), 70.5,72.1 .72 .2,72.5 .73 .0 .96 .6,101.9$. 128.5. 129.0. 129.3, 129.8, 130.0, 133.3, 133.6, 165.3(2). 165.6. $165.8(2), 165.9$. 166.3: IR ( $\mathrm{CHCl}_{3}$ film) $1216,1455$. $1606.173 \mathrm{I} \mathrm{cm}^{-1}$.

Methyl (2,3,4,6-Tetra- $O$-benzyl- $\alpha, \beta$-D-glucopyranosyl)$(1 \rightarrow 6)$-2,3,t-tri-O-benzoyl- $\alpha$-D-glucopyranoside (10). A solution of 2-(benzyloxycarbonyl)benzyl 2,3.4,6-tetra-Obenzyl- $\beta$-D-glucopyranoside (5) $(92 \mathrm{mg}, 0.12 \mathrm{mmol}, 1.0$ equiv), methyl 2,3.4-tri- $O$-benzoyl- $\alpha$-D-glucopyranoside (8) (182 $\mathrm{mg} .0 .36 \mathrm{mmol}, 3.0$ equiv), and TMSOTf ( $65 \mu \mathrm{L} .0 .36$ mmol. 3.0 equiv. ) in $\mathrm{CH}_{3} \mathrm{CN}(5 \mathrm{~mL})$ was stirred at $-25^{\circ} \mathrm{C}$ for $3 \mathrm{~h}$ and the reaction mixture was quenched with saturated aqueous $\mathrm{NaHCO}_{3}$ solution $(2 \mathrm{~mL})$ and then extracted with $\mathrm{CH}_{2} \mathrm{Cl}_{2}(3 \times 20 \mathrm{~mL})$. The combined organic layer was washed with brine $(20 \mathrm{~mL})$, dried $\left(\mathrm{MgSO}_{4}\right)$, and concentrated in vacuo. The residue was purified by silica gel flash column clromatography (toluene/EtOAc. $10: 1 . \mathrm{v} / \mathrm{v}$ ) to afford the disaccharide $\mathbf{1 0}(97 \mathrm{mg}, 78 \%, \alpha: \beta=1 ; 3) . \mathbf{1 0 \alpha}$. colorless oil, $\mathrm{R}_{f}=0.60(n$-hexane/EtOAc, $2: 1 . \mathrm{v} / \mathrm{v}):[\alpha]_{\mathrm{D}}^{3 \mathrm{v}}=$ $+54.0\left(\mathrm{c}=1.1 . \mathrm{CHCl}_{3}\right) ;{ }^{1} \mathrm{H} \mathrm{NMR}\left(250 \mathrm{MHz} . \mathrm{CDCl}_{3}\right) \delta 3.43$ (s. $3 \mathrm{H}) .3 .48-3.66(\mathrm{~m} .5 \mathrm{H}) .3 .8 \mathrm{l}(\mathrm{m}, 2 \mathrm{H}), 3.96$ (dd. $J=9.3$. $9.3 \mathrm{~Hz}, \mathrm{lH}), 4.24-4.28(\mathrm{~m}, \mathrm{lH}) .4 .37(\mathrm{~d} . J=12.3 \mathrm{~Hz} . \mathrm{lH})$. $4.44(\mathrm{~d} . J=11.0 \mathrm{~Hz}, 1 \mathrm{H}) .4 .55(\mathrm{~d} . J=12.3 \mathrm{~Hz}, \mathrm{lH}) .4 .62(\mathrm{~d}$. $J=12.5 \mathrm{~Hz}, \mathrm{lH}) .4 .73$ (s. $2 \mathrm{H}), 4.79(\mathrm{~s} . \mathrm{HH}), 4.81(\mathrm{~d}, J=12.5$ $\mathrm{Hz}, \mathrm{lH}) .4 .9 \mathrm{l}(\mathrm{d}, J=11.0 \mathrm{~Hz}, \mathrm{lH}) .5 .19-5.24(\mathrm{~m}, 2 \mathrm{H}) .5 .52$ (dd. $J=9.7,9.7 \mathrm{~Hz}, 1 \mathrm{H}$ ). 6.13 (dd. $J=9.7,9.7 \mathrm{~Hz}, \mathrm{IH}$ ). $7.11-7.57(\mathrm{~m}, 29 \mathrm{H}), 7.80-8.09(\mathrm{~m}, 6 \mathrm{H}) \cdot{ }^{13} \mathrm{C}$ NMR $(63 \mathrm{MHz}$. $\left.\mathrm{CDCl}_{3}\right) \delta 55.7,66.8,68.4,68.7,69.8,70.4 .70 .8 .72 .4 .73 .3$. 73.5(2) . 74.9. 75.7. 80.1, 82.0.96.9.97.4. 127.6. 127.8(2). 128.0. 128.1, 128.4(2), 128.5(2), 128.7, 129.3, 129.5, 129.9. 130.0, 130.1. 133.2 133.5(2) $138.0,138.5,138.7,139.0$. 165.4. 166.0(2): IR (NaCl) 1101. $1278.1736 \mathrm{~cm}^{-1}$ : Anal. Calcd for $\mathrm{C}_{63} \mathrm{H}_{6 i} \mathrm{O}_{14}:$ C. 72.36: H. 5.88. Found: C. 72.31: H. 5.87. 10B: colorless oil. $\mathrm{R}_{f}=0.65$ (n-hexane/EtOAc. $2: 1$. $\mathrm{v} / \mathrm{v}):[\alpha]_{\mathrm{D}}^{2 \mathrm{n}}=+3.48\left(\mathrm{c}=1.4 . \mathrm{CHCl}_{3}\right):{ }^{l} \mathrm{H}$ NMR $(250 \mathrm{MHz}$. $\left.\mathrm{CDCl}_{3}\right) \delta 3.37$ (s. $3 \mathrm{H}$ ). $3.43-3.71$ (m. 6H). 3.80 (dd $J=7.5$. $10.8 \mathrm{~Hz}, 1 \mathrm{H}), 4.10-4.14(\mathrm{~m} .1 \mathrm{H}) .4 .35-4.55$ (m. $5 \mathrm{H}), 4.66-$ $4.82(\mathrm{~m} .3 \mathrm{H}) .4 .91(\mathrm{~d} . J=10.9 \mathrm{~Hz} .1 \mathrm{H}) .5 .06(\mathrm{~d} . J=10.9 \mathrm{~Hz}$. lH) $5.20-5.32(\mathrm{~m}, 2 \mathrm{H}) .5 .47(\mathrm{dd} . J=9.7 .9 .7 \mathrm{~Hz}, 1 \mathrm{H}) .6 .17$ (dd. $J=9.7 .9 .7 \mathrm{~Hz}, 1 \mathrm{H}) .7 .13-7.53(\mathrm{~m} .29 \mathrm{H}) .7 .83-7.98(\mathrm{~m}$. $6 \mathrm{H}):{ }^{13} \mathrm{C}$ NMR $\left(63 \mathrm{MHz}, \mathrm{CDCl}_{3}\right) \delta 55.6,68.8,69.0 .69 .1$. $70.0 .70 .6 .72 .3,73.6 .74 .9 .75 .0 .75 .1 .75 .8 .77 .8 .82 .5 .84 .7$. 96.9. 104.1. 127.7. 127.8. 127.9. 128.0. 128.1. 128.3. 128.4. 128.5(2). 129.1. 129.2. 129.4, 129.8, 130.0,130.1 133.2. 133.5(2). 138.3. 138.6 138.8, 165.6. 165.9. 166.0: IR $\left(\mathrm{CHCl}_{3}\right.$ film) $2931.1729,1281,1103 \mathrm{~cm}^{-1}$. Anal. Calcd for $\mathrm{C}_{6}: \mathrm{H}_{6 i} \mathrm{O}_{14}:$ C. 72.36: H. 5.88. Found: C. 72.34: H. 5.88 .
Methyl (2,3-Di- $O$-benzoyl-4,6- $O$-cyclohexylidene- $\beta$-Dglucopyranosyl)-(1 $\rightarrow 6)$-2,3,4-tri- $O$-benzoyl- $\alpha$-D-glucopyranoside (11). A solution of 2-(benzyloxycarbonyl)benzyl 2,3-di-O-benzoyl-4,6-O-cyclohexylidene-3-D-glucopyranoside (7) $(50 \mathrm{mg}$. $0.072 \mathrm{mmol} .1 .0$ equiv). methyl 2.3,4-tri-O-benzoyl- $\alpha$-D-glucopyranoside (8) (1 $10 \mathrm{mg}$. 0.22 mmol. 3.0 equiv). and TMSOTf ( $80 \mathrm{mg}$. 0.36 mmol. 5.0 equiv) in $\mathrm{CH}_{2} \mathrm{Cl}_{2}(5 \mathrm{~mL})$ was stirred at $0^{\circ} \mathrm{C}$ for $3 \mathrm{~h}$ and the reaction mixture was quenched with saturated aqueous $\mathrm{NaHCO}_{3}$ solution $\left(2 \mathrm{~mL}\right.$ ) and then extracted with $\mathrm{CH}_{2} \mathrm{Cl}_{2}(3$ $\times 15 \mathrm{~mL}$ ). The combined organic layer was washed with brine $(15 \mathrm{~mL})$, dried $\left(\mathrm{MgSO}_{4}\right)$. and concentrated in vacuo. The residue was purified by silica gel flash column chromatography ( $n$-hexane/EtOAc. $2: 1 . \mathrm{v} / \mathrm{v})$ to afford the disaccharide $11\left(51 \mathrm{mg} .73 \%\right.$ ) as white solids: $\mathrm{mp} 79-82^{\circ} \mathrm{C}$ : $\mathrm{R}_{f}=0.68(n$-hexane/EtOAc. $1: 2 . \mathrm{v} / \mathrm{v}):[\alpha]_{\mathrm{n}}^{20}=+33.5(\mathrm{c}=$ 1.0, $\left.\mathrm{CHCl}_{3}\right)$. ${ }^{1} \mathrm{H}$ NMR $\left(250 \mathrm{MHz}, \mathrm{CDCl}_{3}\right) \delta 1.23-1.69(\mathrm{~m}$, $10 \mathrm{H}) .3 .39$ (s. $3 \mathrm{H}) .3 .74(\mathrm{dd}, J=6.0 .11 .3 \mathrm{~Hz} . \mathrm{lH}), 3.94-4.04$ (m. $2 \mathrm{H}) .4 .24-4.30(\mathrm{~m} . \mathrm{lH}) .4 .39-4.5 \mathrm{l}(\mathrm{m}, \mathrm{lH}) .4 .53(\mathrm{dd} . J=$ $5.4,7.9 \mathrm{~Hz} . \mathrm{lH}), 5.19-5.32(\mathrm{~m}, 3 \mathrm{H}) .5 .54-5.61$ (m. $2 \mathrm{H}) .5 .81$ (dd. $J=1.1,5.3 \mathrm{~Hz} . \mathrm{lH}) .6 .12-6.20(\mathrm{~m}, \mathrm{lH}), 7.23-8.0 \mathrm{l}(\mathrm{m}$. $25 \mathrm{H}):{ }^{13} \mathrm{C}$ NMR $\left(63 \mathrm{MHz}, \mathrm{CDCl}_{3}\right) \delta 23.6 .24 .0,25.2,34.6$. 36.5 .55 .7 .66 .8 . 669.99 .2 .69 .5 .70 .7 .72 .3 .73 .7 .74 .8 .81 .0 . $82.7,97.0 .106 .7$. 110.0. 128.4. 128.5, 128.6, 129.1, 129.2, 129.4. 129.8. 130.0(2). 133.2, 133.5, 133.6, 165.0, 165.2. 165.3, 165.9(2): IR ( $\mathrm{CHCl}_{2}$ film) l104. 1275. 1460, 1736 $\mathrm{cm}^{-1}$. Anal. Calcd for $\mathrm{C}_{54} \mathrm{H}_{5} \mathrm{O}_{16}: \mathrm{C}, 67.77: \mathrm{H}, 5.48$. Found: C. 67.76: H, 5.44.

Acknowledgement. This work was supported by Korea Research Foundation Grant (KRF-2001-042-D00049). Authors thank CBMH.

\section{References}

1. (a) Varki. A. Ghycobiologv 1993. 3. 97, (b) Dwek. R. A. Chem. Rev: 1996. 96, 683. Bertozzi C. R.: Kiessling. L. L. Science 2001. 291.2357

2. (a) Toshima, K.; Tatsuta, K. Chem. Rev 1993. 93,1503 . (b) Davis. B. G. J. Chent. Soc., Perkin Trans 12000.2137.

3. (a) Plante. O. J.: Palmacci. E. R.: Andrade. R. B.: Seeberger. P. H. J. Am. Chem. Soc. 2001. 123.9545. (b) Hinklin. R. J.: Kiessling. L. L. J. Am. Chem. Soc. 2001, 123, 3379. (c) Davis. B. J.: Ward, S. J: Rendle, P. M. Chem Commun 2001, 189. (d) Petersen. L: Jensen, K. J. J. Org. Chem. 2001. 66.6268. (e) Garcia. B. A.: Gin. D. Y. J. Am. Chem. Soc. 2000. 122. 4269. (f) Nguyen. H. M: Chent. Y.: Duron. S. G.: Gin. D. Y. J. An. Chem. Soc. 2001.123. 8766. (g) Crich. D.: Sun. S. J. Am. Chem. Soc. 1998. 120.435. (h) Crich. D.: Smith. M. J. Am. Chem. Soc. 2001. 123. 9015.

4. Kim. K. S.: Kim, J. H.: Lee, Y. J : Lee, Y. J.: Park. J. J. Am. Chem. Soc. $2001,123,8477$.

5. Kim. K. S.: Park. J.: Lee. Y. J.: Seo. Y. S. Angerv. Chem. In. Ed. 2003. +2.459

6. Kim. K. S.: Kang. S. S.: Seo. Y. S.: Kim. H. J.: Lee. Y. J.: Jeong K.-S. Synlett 2003. 1311.

7. Fletcher, H. G. In Hethods in Carbohydrate Chemisny. Whistler. R. L.; Wolfrom. M. L. Eds.: Academic Press: New York. U. S. A.. 1963: Vol. 2. p 226.

8. Mootoo. D. R.: Konradssont. P.: Udodong. U.: Fraser-Reid. B. $J$. Am. Chem. Soc. 1988. 110. 5583.

9. Yamago. S.: Kolubo. K Hara, O.; Masuda, S.; Yoshida. J. J. Org. Chem. 2002, 67, 8584 . 